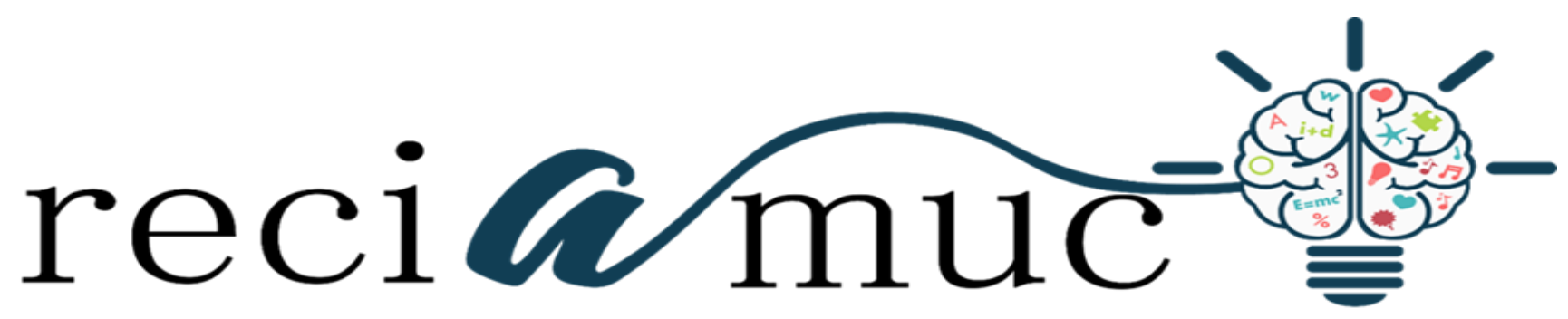

Revista cientifica de investigación actualización del mundo de las ciencias

Edwin José Loor Sánchez a ; Jennifer Estefania Bailón Mieles b; Angélica Cristina

López Alcívar c; María Elisa Avendaño Robles d; Francel Omar Campoverde Imbaquingo e; Andrea Krusenka Rivas Loor ${ }^{\mathrm{f}}$

Prevención y cuidados post operatorios de las mujeres sometidas a cirugía de cáncer de mama

Prevention and post-operative care of women undergoing breast cancer surgery

Revista Científica de Investigación actualización del mundo de las Ciencias. Vol. 3 núm., 3, julio, ISSN: 2588-0748, 2018, pp. 1429-1443

DOI: $10.26820 /$ reciamuc/3.(3).julio.2019.1429-1443

URL: http://reciamuc.com/index.php/RECIAMUC/article/view/398

Código UNESCO: 3205 Medicina Interna

Tipo de Investigación: Artículo de Investigación

(C) RECIAMUC; Editorial Saberes del Conocimiento, 2019

Recibido: 28/04/2019

Aceptado: 19/05/2019

Publicado: 01/07/2019

Correspondencia: dr.edwinloorsanchez@hotmail.com

a. Médico; Hospital General IESS; Manta - Ecuador; dr.edwinloorsanchez@hotmail.com

b. Médico; Hospital General IESS; Manta - Ecuador; jenniferestefania510@outlook.com

c. Médico Cirujano; Médico General en Funciones hospitalarias IESS; Manta - Ecuador; angeleslopez1427@gmail.com

d. Médica Cirujana; Hospital General IESS; Manta - Ecuador; eligattis@ hotmail.com

e. Médico; Clínica Santa Bárbara - Área de hospitalización Clínica, Quirúrgica y Pediatría; Quito - Ecuador; francelcampoverde8508@ hotmail.es

f. Médica Cirujana; Investigadora Independiente; Portoviejo - Ecuador; drakrusenka@gmail.com 


\section{Prevención y cuidados post operatorios de las mujeres sometidas a cirugía de cáncer de mama}

Vol. 3, núm. 3., (2019)

Edwin José Loor Sánchez; Jennifer Estefania Bailón Mieles; Angélica Cristina López Alcívar; María Elisa Avendaño Robles; Francel Omar Campoverde Imbaquingo; Andrea Krusenka Rivas Loor

\section{RESUMEN}

La prevención del cáncer consiste en practicar y conocer las medidas que se toman para reducir la probabilidad de desarrollar esta enfermedad. Algunos factores de riesgo del cáncer de seno relacionados con el estilo de vida: consumo de bebidas alcohólicas, sobrepeso u obesidad, inactividad física, mujeres que no han tenido hijos, no dar de lactar a sus bebés, el uso de anticonceptivos orales, la inyección anticonceptiva, los implantes, dispositivos intrauterinos, parches cutáneos, anillos vaginales para el control de la natalidad, terapia hormonal después de la menopausia. Desde el punto de vista preventivo, el ejercicio y una dieta equilibrada disminuyen el riesgo de padecer cáncer de mama. Para las pacientes ya diagnosticadas se sugiere que el ejercicio disminuye el riesgo de recaída. Dada de alta hospitalaria la paciente debe cumplir las pautas de medicación que le prescriba su cirujano: analgésicos, antiinflamatorios y en ocasiones relajantes musculares y heparina. No debe mojar las incisiones, preservándolas limpias y pinceladas con algún antiséptico de manera frecuente. No fume al menos, hasta que no se hayan retirado las suturas. Durante las primeras 2 o 3 semanas debe llevar una vida tranquila, sin realizar esfuerzos físicos, minimizando el movimiento de los brazos. Podrá hacer vida normal al mes de la intervención, aunque depende en gran medida del tipo de cirugía y de la evolución de cada paciente. Su cirujano debe verla un mínimo de 3 veces en el postoperatorio inmediato: una a la semana, a los 15 días y después al mes. Las revisiones durante el primer año suelen ser a los 3, 6 y 12 meses. La metodología usada es descriptiva, con un enfoque documental, es decir, revisar fuentes disponibles en la red, como google académico, con contenido oportuno y relevante desde el punto de vista científico que enriquezca el análisis del tema planteado en este artículo.

Palabras Claves: Prevención; Ejercicio; Alimentación adecuada a su Salud; Autoexamen; Reposo; Vida Plena; Vigilancia; Asistencia Médica. 


\title{
Prevención y cuidados post operatorios de las mujeres sometidas a cirugía de cáncer de mama
}

Vol. 3, núm. 3., (2019)

Edwin José Loor Sánchez; Jennifer Estefania Bailón Mieles; Angélica Cristina López Alcívar; María Elisa Avendaño Robles; Francel Omar Campoverde Imbaquingo; Andrea Krusenka Rivas

Loor

\begin{abstract}
Cancer prevention consists in practicing and knowing the measures that are taken to reduce the probability of developing this disease. Some risk factors for breast cancer related to lifestyle: consumption of alcoholic beverages, overweight or obesity, physical inactivity, women who have not had children, not breastfeeding their babies, the use of oral contraceptives, injection contraceptive, implants, intrauterine devices, skin patches, vaginal rings for birth control, hormone therapy after menopause. From a preventive point of view, exercise and a balanced diet reduce the risk of breast cancer. For patients already diagnosed, it is suggested that exercise reduces the risk of relapse. Upon discharge from the hospital, the patient must comply with the medication guidelines prescribed by her surgeon: analgesics, anti-inflammatories and sometimes muscle relaxants and heparin. You should not wet the incisions, keeping them clean and brushing with an antiseptic frequently. Do not smoke at least, until the sutures have been removed. During the first 2 or 3 weeks you should lead a quiet life, without physical efforts, minimizing the movement of the arms. You can live a normal life one month after the intervention, although it depends largely on the type of surgery and the evolution of each patient. Your surgeon should see it a minimum of 3 times in the immediate postoperative period: once a week, 15 days and then a month. Revisions during the first year are usually at 3, 6 and 12 months. The methodology used is descriptive, with a documentary approach that is, reviewing sources available on the web, such as google scholar, with timely and scientifically relevant content that enriches the analysis of the topic raised in this article.
\end{abstract}

Key Words: Prevention; Exercise; Adequate food for your Health; Self-examination; Repose; Full life; Surveillance; Medical assistance. 


\section{Prevención y cuidados post operatorios de las mujeres sometidas a cirugía de cáncer de mama}

Vol. 3, núm. 3., (2019)

Edwin José Loor Sánchez; Jennifer Estefania Bailón Mieles; Angélica Cristina López Alcívar; María Elisa Avendaño Robles; Francel Omar Campoverde Imbaquingo; Andrea Krusenka Rivas Loor

\section{Introducción.}

Un factor de riesgo es todo aquello que aumenta la probabilidad de que una persona desarrolle cáncer. Si bien los factores de riesgo con frecuencia influyen en el desarrollo del cáncer, la mayoría no provocan cáncer de forma directa. Algunas personas con varios factores de riesgo nunca desarrollan cáncer, mientras que otras personas sin factores de riesgo conocidos desarrollan cáncer. Conocer los factores de riesgo, buscar asesoría médica al respecto puede ayudarlo a tomar decisiones sobre las opciones de estilo de vida y atención médica.

La mayoría de los casos de cáncer de mama se desarrollan a partir del daño a los genes de una persona que se produce por casualidad después del nacimiento. No hay riesgo de transmitir este gen a los hijos. Los casos de cáncer de mama hereditario aparecen cuando se transmiten cambios genéticos, llamados mutaciones, dentro de una familia, del padre o la madre al hijo. Muchas de estas mutaciones se encuentran en los genes de supresión de tumores, tales como el BRCA1o el BRCA2. Estos genes evitan que las células crezcan fuera de control y se transformen en cáncer. Pero cuando dichas células presentan una mutación, pueden desarrollarse sin control.

Al considerar el riesgo de desarrollar cáncer de mama, es importante recordar que la mayoría de las mujeres que desarrollan cáncer de mama no presentan factores de riesgo evidentes ni antecedentes familiares de cáncer de mama. Múltiples factores de riesgo influyen en el desarrollo del cáncer de mama. Esto significa que todas las mujeres deben ser conscientes de los cambios en sus mamas. También deben hablar con sus médicos sobre la posibilidad de someterse a exámenes de mama regulares.

La operación no garantiza un éxito total, pero si un avance, dentro de los cuidados post operatorios el paciente debe seguirlos con disciplina, alcanzar la mejoría esperada e incorporar una serie de cambios en el estilo de vida de la mujer y posiblemente a su entorno familiar como se explicará posteriormente en el presente artículo. La calidad y disponibilidad de la información oportuna permite al paciente hacer la diferencia en la aceptación de una enfermedad difícil de llevar sin el acompañamiento requerido. 


\section{Prevención y cuidados post operatorios de las mujeres sometidas a cirugía de cáncer de mama}

Vol. 3, núm. 3., (2019)

Edwin José Loor Sánchez; Jennifer Estefania Bailón Mieles; Angélica Cristina López Alcívar; María Elisa Avendaño Robles; Francel Omar Campoverde Imbaquingo; Andrea Krusenka Rivas

Loor

\section{Metodología.}

Esta investigación está dirigida al estudio del "Prevención y cuidados post operatorios de las mujeres sometidas a cirugía de cáncer de mama”. Para realizarlo se usó una metodología tipo descriptiva, con un enfoque documental, es decir, revisar fuentes disponibles en la red, como google académico, con contenido oportuno y relevante desde el punto de vista científico para dar respuesta a lo tratado en el presente artículo y que sirvan de inspiración para realizar otros proyectos. Las mismas pueden ser consultadas al final, en la bibliografía.

\section{Resultados.}

La prevención del cáncer consiste en practicar y conocer las medidas que se toman para reducir la probabilidad de enfermar de cáncer. Con la prevención, se reduce el número de casos nuevos en un grupo o población. Los científicos analizan los factores de riesgo y los factores de protección para prevenir el inicio de cánceres nuevos. Cualquier cosa que aumenta su riesgo de tener cáncer se llama factor de riesgo de cáncer; cualquier mecanismo que disminuye su riesgo de desarrollarlo se llama factor de protección de cáncer.

Aunque algunos factores de riesgo de cáncer se pueden evitar, no todos son controlables, por ejemplo, tanto fumar como heredar ciertos genes son factores de riesgo de algunos tipos de cáncer, pero solo se puede evitar fumar. Hacer ejercicio con regularidad y consumir una alimentación saludable son factores de protección para algunos tipos de cáncer. Es posible que, al evitar los factores de riesgo y aumentar los factores de protección disminuya su riesgo; sin embargo, esto no significa que no padecerás cáncer.

Factores de riesgo del cáncer de seno relacionados con el estilo de vida.

- Consumo de bebidas alcohólicas: las mujeres que toman de dos a tres bebidas al día tienen alrededor de $20 \%$ más riesgo comparado con las mujeres que no toman alcohol

- Sobrepeso u obesidad: después de la menopausia (cuando los ovarios dejan de producir estrógeno), la mayor parte del estrógeno de una mujer proviene del tejido adiposo. Un 


\section{Prevención y cuidados post operatorios de las mujeres sometidas a cirugía de cáncer de mama}

Vol. 3, núm. 3., (2019)

Edwin José Loor Sánchez; Jennifer Estefania Bailón Mieles; Angélica Cristina López Alcívar; María Elisa Avendaño Robles; Francel Omar Campoverde Imbaquingo; Andrea Krusenka Rivas Loor

exceso de tejido adiposo después de la menopausia puede elevar los niveles de estrógeno y aumenta su probabilidad de padecer cáncer de seno.

- Inactividad física: la actividad física está relacionada con la reducción en el riesgo de cáncer de seno, especialmente en las mujeres que han pasado por la menopausia.

- Mujeres que no han tenido hijos: o las que tuvieron su primer hijo después de los 30 años tienen en general un riesgo ligeramente mayor de padecer cáncer de seno.

- No dar de lactar a sus bebés: estudios sugieren que la lactancia podría disminuir ligeramente el riesgo de cáncer de seno, especialmente si se prolonga por $1 \frac{1}{2}$ a 2 años. Control de la natalidad: las mujeres que usan anticonceptivos orales tienen un riesgo ligeramente mayor de tener cáncer de seno que aquellas mujeres que nunca los han usado. La inyección anticonceptiva, los implantes, dispositivos intrauterinos, parches cutáneos, anillos vaginales para el control de la natalidad: son métodos de control de la natalidad que utilizan hormonas, podrían impulsar el crecimiento del cáncer de seno.

- Terapia hormonal después de la menopausia: la decisión de usar la terapia hormonal debe tomarla la mujer y su médico después de analizar los posibles riesgos y beneficios (incluyendo la gravedad de los síntomas de la menopausia), y considerar los otros factores de riesgo de enfermedad cardiaca, cáncer de seno y osteoporosis. Si deciden emplear la terapia hormonal como tratamiento de los síntomas menopáusicos, por lo general es mejor usar la menor dosis posible y por el tiempo más breve que sea eficaz para ella.

Desde el punto de vista preventivo, el ejercicio y una dieta equilibrada disminuyen el riesgo de padecer cáncer de mama. Para las pacientes ya diagnosticadas se sugiere que el ejercicio disminuye el riesgo de recaída. El ejercicio debe ser más intenso que caminar. Hasta hace poco se recomendaba caminar de tres a cuatro días a la semana, pero se descubrió recientemente que el ejercicio más intenso aporta más beneficio. Ahora se recomienda una combinación de ejercicio aeróbico, como correr o hacer bicicleta, con ejercicios de tonificación muscular, como pilates, yoga o ejercicios con pesas con supervisión (Callejo, 2018). 


\section{Prevención y cuidados post operatorios de las mujeres sometidas a cirugía de}

cáncer de mama

Vol. 3, núm. 3., (2019)

Edwin José Loor Sánchez; Jennifer Estefania Bailón Mieles; Angélica Cristina López Alcívar; María Elisa Avendaño Robles; Francel Omar Campoverde Imbaquingo; Andrea Krusenka Rivas

Loor

El ejercicio físico beneficia tanto a las pacientes que están en tratamiento con quimioterapia como a las que reciben terapia hormonal. Las primeras sufren una astenia a veces devastadora por el tratamiento quimioterápicos, el ejercicio les ayuda a superar ese cansancio, se sienten más activas. Las pacientes que ya han terminado el tratamiento o que reciben exclusivamente terapia hormonal también logran mejoría de los problemas musculares y articulares derivados de ella.

Para conocer los ejercicios recomendados a las pacientes y supervivientes de cáncer de mama debe consultar con su médico, la posibilidad de comenzar un programa de ejercicio físico, supervisado por profesionales del ejercicio. Si existe linfedema o no, los efectos secundarios de los tratamientos farmacológicos, la condición física de cada paciente y, por supuesto, de las preferencias de cada uno. La recomendación es la de realizar actividades que involucren grandes grupos musculares, como el ejercicio aeróbico como caminar, nadar, pedalear, etc.

Entre este grupo de actividades tiene especial interés la marcha nórdica. También los ejercicios de fuerza (para fortalecer los músculos) y de flexibilidad (para mejorar el rango de movimiento). Lo ideal es combinar ejercicio aeróbico, más intenso que caminar con ejercicios de fuerza y flexibilidad, como pueden ser pilates o yoga, tres o cuatro días por semana el ejercicio aeróbico y dos días pilates o yoga, por ejemplo.

Cirugía para reducir el riesgo de cáncer: en el caso de las mujeres que presentan mutaciones genéticas del BRCA1 o el BRCA2, que aumentan el riesgo del cáncer de mama, puede considerarse la extirpación preventiva de las mamas. La mastectomía profiláctica reduce el riesgo de desarrollar cáncer de mama en, al menos, el 95\% (American Society of Clinical Oncology, 2018). Su médico le explicará los posibles efectos secundarios físicos y emocionales cuando considere la posibilidad de someterse a estos procedimientos.

Fármacos para disminuir el riesgo de cáncer: las mujeres con un riesgo elevado de desarrollar cáncer de mama pueden evaluar determinados fármacos que podrían ayudar a prevenir el cáncer de mama, se trata del uso de fármacos de bloqueo hormonal para reducir el riesgo de cáncer. Los 


\section{Prevención y cuidados post operatorios de las mujeres sometidas a cirugía de cáncer de mama}

Vol. 3, núm. 3., (2019)

Edwin José Loor Sánchez; Jennifer Estefania Bailón Mieles; Angélica Cristina López Alcívar; María Elisa Avendaño Robles; Francel Omar Campoverde Imbaquingo; Andrea Krusenka Rivas Loor

fármacos tamoxifeno y raloxifeno están aprobados por la Administración de Alimentos y Medicamentos para reducir el riesgo de cáncer de mama. Estos fármacos se llaman moduladores selectivos de los receptores estrogénicos y no son quimioterapia.

Un Serm es un medicamento que bloquea los receptores de estrógeno en algunos tejidos y no en otros. Tanto las mujeres que han transitado la menopausia como aquellas que no pueden tomar el tamoxifeno. El raloxifeno solo está aprobado para mujeres que han transitado la menopausia. Cada fármaco también produce efectos secundarios Los inhibidores de la aromatasa también demostraron reducir el riesgo de cáncer de mama. Los AI son un tipo de tratamiento de bloqueo hormonal que reduce la cantidad de estrógeno en el cuerpo de la mujer impidiendo que tejidos y órganos diferentes de los ovarios produzcan estrógeno. Solo pueden utilizarse en mujeres que han transitado la menopausia. Otros fármacos que se están estudiando para reducir el riesgo de cáncer de mama incluyen las estatinas, que reduce el colesterol, y la metformina, un fármaco frecuentemente utilizado para la diabetes

Los factores reproductivos asociados a una exposición prolongada a estrógenos endógenos, como una menarquia precoz, una menopausia tardía y una edad madura para el primer parto figuran entre los factores de riesgo más importantes del cáncer de mama. Las hormonas exógenas también conllevan un mayor riesgo de cáncer de mama, las usuarias de anticonceptivos orales y de tratamientos de sustitución hormonal tienen más riesgo que las mujeres que no usan esos productos. La lactancia materna por el contrario, tiene un efecto protector (A. Danaei, 2009). Los autores concluyen que el $21 \%$ de todas las muertes por cáncer de mama registradas en el mundo son atribuibles al consumo de alcohol, el sobrepeso y la obesidad, y la falta de actividad física.

Esa proporción fue mayor en los países de ingresos altos (27\%), y el factor más importante fue el sobrepeso y la obesidad. En los países de ingresos bajos y medios, la proporción de cánceres de mama atribuibles a esos factores de riesgo fue del 18\%, y la falta de actividad física fue el factor determinante más importante (10\%). La diferente incidencia del cáncer de mama en los países desarrollados y los países en desarrollo puede explicarse en parte por los efectos de la 


\section{Prevención y cuidados post operatorios de las mujeres sometidas a cirugía de cáncer de mama}

Vol. 3, núm. 3., (2019)

Edwin José Loor Sánchez; Jennifer Estefania Bailón Mieles; Angélica Cristina López Alcívar; María Elisa Avendaño Robles; Francel Omar Campoverde Imbaquingo; Andrea Krusenka Rivas

Loor

alimentación, unidos a la mayor edad del primer embarazo, el menor número de partos y el acortamiento de la lactancia (Peto, 2001). La creciente adopción de modos de vida occidental en los países de ingresos bajos y medios es un determinante importante del incremento de la incidencia de cáncer de mama en esos países.

\section{Cuidados post operatorios.}

Finalizada la cirugía de la mama con anestesia general es recomendable permanecer hospitalizada por 24 horas, con el cabecero levemente inclinado para impedir que la paciente dormida se tumbe de lado. Esta posición mejora el drenaje de los tejidos mamarios y reduce la inflamación. La paciente no debe realizar movimientos bruscos con los brazos, aunque no sienta dolor. El no guardar reposo así la paciente no tenga molestia alguna, puede desencadenar complicaciones como rotación de la prótesis en caso de mamoplastias de aumento, o hemorragias en cualquier tipo de intervención, aumento el riesgo de cirugías secundarias. En caso de una reconstrucción con colgajos libres o pediculados, se debe evitar comprimir toda la zona intervenida para no colapsar el pedículo que vasculariza el colgajo.

Dada de alta hospitalaria la paciente debe cumplir exhaustivamente las pautas de medicación que le mande su cirujano, esencialmente analgésicos, antiinflamatorios y en ocasiones relajantes musculares y heparina. No debe mojar las incisiones, preservándolas limpias y pinceladas con algún antiséptico de manera frecuente. No fume al menos, hasta que no se hayan retirado las suturas, siendo ideal el cese completo del hábito tabáquico. En caso de necesitar alguna prenda de presoterapia como sujetador ortopédico o fajas, debe usarlos las 24 horas del día durante el primer mes para prevenir el desarrollo de seromas y para fijar las cápsulas en caso de cirugías que incluyan prótesis. Sólo deberá quitarse estas prendas para la higiene diaria.

Durante las primeras 2 o 3 semanas debe llevar una vida tranquila, sin realizar esfuerzos físicos, minimizando el movimiento de los brazos. Podrá hacer vida normal al mes de la intervención, aunque depende en gran medida del tipo de cirugía y de la evolución de cada paciente. Su cirujano debe verla un mínimo de 3 veces en el postoperatorio inmediato: una a la semana, a los 


\section{Prevención y cuidados post operatorios de las mujeres sometidas a cirugía de cáncer de mama}

Vol. 3, núm. 3., (2019)

Edwin José Loor Sánchez; Jennifer Estefania Bailón Mieles; Angélica Cristina López Alcívar; María Elisa Avendaño Robles; Francel Omar Campoverde Imbaquingo; Andrea Krusenka Rivas Loor

15 días y después al mes. Las revisiones durante el primer año suelen ser a los 3, 6 y 12 meses. En caso de aparecer cualquier eventualidad o complicación fuera de las fechas de revisión, no dude de ponerse en contacto inmediato con su cirujano (López, 2017).

Algunas instrucciones y medidas, relativas a los cuidados del brazo después de la extirpación de estos ganglios que son esenciales para recuperar la movilidad del hombro y prevenir posibles complicaciones posteriores como limitación de la amplitud de movimientos, infecciones del brazo, edema, etc.

Medidas Higiénicas_(Clínica Universitaria de Navarra, 2007)

- Debe procurar, mientras esté sentada, mantener el brazo apoyado en alto.

- Evite llevar el brazo colgando, especialmente con algún peso.

- Procure no dormir sobre el brazo operado.

- Evite cualquier sobrecarga o esfuerzo con ese brazo.

- Cuide de forma meticulosa la piel (jabón neutro, cremas hidratantes...).

- Procure depilarse con crema o de forma eléctrica.

- Debe ser cuidadosa al cortarse las uñas y hacer la manicura.

- Evite la aplicación de irritantes (colonias, desodorantes con alcohol, etc.).

- No utilice objetos que compriman (anillos, pulseras, reloj, mangas prietas).

- Utilice un sujetador de tirante ancho, evitando la presión sobre el hombro.

- Evite las extracciones de sangre en ese brazo.

- Utilice dedal al coser.

- Evite el calor sobre el brazo: sol, horno, plancha, agua caliente, etc.

Acuda a su médico tratante si observa alguno de estos síntomas (Alvarado, 2013): 


\section{Prevención y cuidados post operatorios de las mujeres sometidas a cirugía de cáncer de mama}

Vol. 3, núm. 3., (2019)

Edwin José Loor Sánchez; Jennifer Estefania Bailón Mieles; Angélica Cristina López Alcívar; María Elisa Avendaño Robles; Francel Omar Campoverde Imbaquingo; Andrea Krusenka Rivas

Loor

- Signos de infección, incluso fiebre y escalofrío por más de 24 horas.

- Enrojecimiento, inflamación, dolor, sangrado excesivo o algún tipo de secreción.

- Tos, falta de aire, dolor en el pecho, nausea o vómito intenso.

- Enrojecimiento, calor, rigidez o endurecimiento del brazo de donde se extrajeron los ganglios linfáticos.

- Bultos o cambios en la piel de los tejidos circundantes del área operada.

\section{Ecuador en cifras.}

Para el año 2017, la Sociedad de Lucha contra el Cáncer (Solca) Guayaquil registró 751 casos de cáncer de mama, que constituyeron 186 casos más respecto al 2016 cuando se contabilizaron 565. En el primer semestre del 2018, la cifra fue de 369, autoridades en la materia no considera que la enfermedad esté en aumento, sino que más mujeres están preocupadas en realizarse chequeos (Sociedad de Lucha contra el Cancer, 2018).

Toda mujer de 40 años debe practicarse una ecosonografía mamaria, según los protocolos a nivel mundial, si el médico detecta factores de riesgo hereditario o conocido, este debe enviar a la paciente a hacerse una resonancia magnética nuclear. La autoexploración como medida preventiva, si hay sospecha de que la forma de las mamas es anormal, si existen molestias, dolor, para la prevención de este cáncer, que junto con el cervicouterino son los de mayor índice en la población femenina.

El cáncer es una enfermedad que avanza con rapidez, por eso mientras más temprano podemos detectarlo será mejor para el paciente, en la jornada del 13 de octubre de 2017, efectuada en la explanada de Mall El Fortín, 2.500 mujeres accedieron a exámenes gratuitos de mama, el apoyo 


\section{Prevención y cuidados post operatorios de las mujeres sometidas a cirugía de cáncer de mama}

Vol. 3, núm. 3., (2019)

Edwin José Loor Sánchez; Jennifer Estefania Bailón Mieles; Angélica Cristina López Alcívar; María Elisa Avendaño Robles; Francel Omar Campoverde Imbaquingo; Andrea Krusenka Rivas Loor

de empresas comprometidas con la sociedad, permite costear mamografías y ecografías para que no tengan costo para la paciente.

Las estadísticas de la Organización Mundial de la Salud (OMS) revelan importantes diferencias en la incidencia y la mortalidad del cáncer de mama en países de América Latina y en España. Las cifras más negativas o de peor desempeño la tienen España, Argentina y Uruguay. República Dominicana no tiene puesto en marcha ningún tratamiento de detección precoz en el sistema de salud pública como las mamografías. Latinoamérica sigue en deuda en la lucha contra el cáncer de mama debido a la falta de un diagnóstico y tratamiento oportuno, la poca educación de las pacientes, los altos costos de una mastografía y la baja inversión en salud, dijo a Efe el doctor Carlos Domínguez, director general médico de la Fundación de Cáncer de Mama (Fucam) (Rodriguez, 2018).

En América Latina tan solo un 60\% sobrevive al cáncer de mama, en países europeos se ha demostrado que si se tiene cobertura de $70 \%$ de población se puede disminuir en 30\% mortalidad de cáncer de mama y es algo que en Latinoamérica no se ha logrado aún. No hay forma de evitar la enfermedad, pero se puede tratar si se detecta oportunamente y las pacientes pueden tener una sobrevida de entre el 98 y el 100\% (Rodriguez, 2018). Aunque España tiene la incidencia más alta de todos los países analizados, tiene una de las mortalidades más bajas.

De acuerdo con datos de la Organización Panamericana de la Salud (OPS), se estima que 30\% de la población en América Latina no tiene acceso a atención de salud debido a razones económicas y que $21 \%$ renuncia a buscar atención debido a las barreras geográficas. Es importante conocer los factores de riesgo como no tener un hijo a término antes de los 30 años, haber empezado a reglar antes de 11 años y dejar de hacerlo después de los 50, ingerir hormonas en la menopausia y tener familiares con cáncer de mama (Organización Mundial de la Salud (OMS), 2017).

\section{Conclusión.}




\section{Prevención y cuidados post operatorios de las mujeres sometidas a cirugía de}

cáncer de mama

Vol. 3, núm. 3., (2019)

Edwin José Loor Sánchez; Jennifer Estefania Bailón Mieles; Angélica Cristina López Alcívar; María Elisa Avendaño Robles; Francel Omar Campoverde Imbaquingo; Andrea Krusenka Rivas

Loor

La prevención del cáncer consiste en practicar y conocer medidas que se toman para reducir la probabilidad de enfermar de cáncer. Con la prevención, se reduce el número de casos nuevos en un grupo o población. Los factores de riesgo y los factores de protección para prevenir el inicio de cánceres nuevos han evolucionado gracias a la tecnología y centros diagnósticos de salud, cercanos a la población más vulnerable. Cualquier elemento que aumenta su riesgo de tener cáncer se llama factor de riesgo; cualquier mecanismo que disminuye su riesgo de desarrollarlo se llama factor de protección de cáncer.

Cambios en el estilo de vida en la paciente durante el tratamiento y posterior a la intervención quirúrgica beneficiaran la calidad de vida de la paciente como: incluir el ejercicio en su rutina, mantener un peso saludable, disminuir la ingesta de azucares refinada y grasas saturadas, incorporar alimentos más verdes y menos procesados, concordancia a la edad y peso de la paciente, eliminar el consumo de cigarrillo, la evaluación médica anual oportuna, por mencionar pequeñas acciones con grandes beneficios en materia de salud.

Tras el alta hospitalaria la paciente debe cumplir exhaustivamente las pautas de medicación que le sugiera su cirujano, esencialmente analgésicos, antiinflamatorios y en ocasiones relajantes musculares y heparina. No debe mojar las incisiones, preservándolas limpias y pinceladas con algún antiséptico de manera frecuente. Durante las primeras 2 o 3 semanas debe llevar una vida tranquila, sin realizar esfuerzos físicos, minimizando el movimiento de los brazos. Podrá hacer vida normal al mes de la intervención, aunque depende en gran medida del tipo de cirugía y de la evolución de cada paciente. Su cirujano debe verla un mínimo de 3 veces en el postoperatorio inmediato: una a la semana, a los 15 días y después al mes. Las revisiones durante el primer año suelen ser a los 3, 6 y 12 meses. En caso de aparecer cualquier eventualidad o complicación fuera de las fechas de revisión, no dude de ponerse en contacto inmediato con su cirujano.

En América Latina una parte de los casos sobrevive al cáncer de mama, en países europeos se ha demostrado que si aumentas cobertura los centros de salud y diagnósticos a la población se puede disminuir la mortalidad de cáncer de mama, en Latinoamérica no se ha logrado aún. No hay forma de evitar la enfermedad, pero se puede tratar si se detecta oportunamente y las 


\section{Prevención y cuidados post operatorios de las mujeres sometidas a cirugía de cáncer de mama}

Vol. 3, núm. 3., (2019)

Edwin José Loor Sánchez; Jennifer Estefania Bailón Mieles; Angélica Cristina López Alcívar; María Elisa Avendaño Robles; Francel Omar Campoverde Imbaquingo; Andrea Krusenka Rivas Loor

pacientes pueden tener una sobrevida, buena parte de la población en América Latina no tiene acceso a atención de salud debido a razones económicas, otras mujeres renuncian a buscar atención debido a las barreras geográficas. Es importante conocer los factores de riesgo como no tener un hijo a término antes de los 30 años, haber empezado a reglar antes de 11 años y dejar de hacerlo después de los 50, ingerir hormonas en la menopausia.

\section{Bibliografía.}

A. Danaei. (Abril de 2009). Cáncer de mama: prevención y control. Obtenido de Organización Mundial de la Salud OMS: www.who.int

Alvarado, Y. (16 de Julio de 2013). Cuidados despues de una mastectomia. Obtenido de MEDICARTE: http://www.medicarteoncologia.com

American Society of Clinical Oncology. (20 de Octubre de 2018). Cáncer de mama: Factores de riesgo y prevención. Obtenido de Cancer.Net: https://www.cancer.net.es

Callejo, A. (19 de Octubre de 2018). Ejercicios más adecuados tras el cáncer de mama. Obtenido de CUIDATEPLUS: www.cuidateplus.com

Clínica Universitaria de Navarra. (08 de Mayo de 2007). Cuidados después de cirugía de la mama y de la axila. Obtenido de Pfizer: https://www.pfizer.es

López, G. (26 de Diciembre de 2017). Post operatorio: claves para una rápida y adecuada recuperación. Obtenido de Cirugías de la Mama: www.cirugiasdelamama.com

Organización Mundial de la Salud (OMS). (05 de Octubre de 2017). El cáncer de mama es la principal causa de muerte en América Latina. Obtenido de EFE Noticias: https://www.diariolasamericas.com

Rodriguez, M. (19 de Octubre de 2018). La deuda de América Latina con el cáncer de mama en cifras. Obtenido de EURONEWS: https://es.euronews.com 
Prevención y cuidados post operatorios de las mujeres sometidas a cirugía de cáncer de mama

Vol. 3, núm. 3., (2019)

Edwin José Loor Sánchez; Jennifer Estefania Bailón Mieles; Angélica Cristina López Alcívar; María Elisa Avendaño Robles; Francel Omar Campoverde Imbaquingo; Andrea Krusenka Rivas Loor

Sociedad de Lucha contra el Cancer. (22 de Octubre de 2018). Ecuador: casos de cancer de mama se incrementaron en un año en Guayaquil. Obtenido de Clustersalud: https://clustersalud.americaeconomia.com

$$
\text { (c) } \frac{1}{\mathrm{BY}(\mathrm{NC}) \mathrm{SA}}
$$

RECONOCIMIENTO-NOCOMERCIAL-COMPARTIRIGUAL

CC BY-NC-SA

ESTA LICENCIA PERMITE A OTROS ENTREMEZCLAR, AJUSTAR Y CONSTRUIR A PARTIR DE SU OBRA CON FINES NO COMERCIALES, SIEMPRE Y CUANDO LE RECONOZCAN LA AUTORÍA Y SUS NUEVAS CREACIONES ESTÉN BAJO UNA LICENCIA CON LOS MISMOS TÉRMINOS. 